\title{
Publicaciones científicas estudiantiles producidas en el curso de Epidemiología de la Facultad de Medicina de la Universidad Nacional Mayor de San Marcos durante el periodo 2003-2009
}

\author{
Medical students' publications produced in the Epidemiology course of the Faculty of Medi- \\ cine, San Marcos University during the period 2003-2009
}

\author{
Jorge Alarcón-Villaverde ${ }^{1,2}$, Franco Romaní2, César Gutiérrez ${ }^{1,2}$ \\ ${ }^{1}$ Departamento Académico de Medicina Preventiva y Salud Pública, Facultad de Medicina, Universidad Nacional Mayor de San Marcos. Lima, Perú. \\ ${ }^{2}$ Sección de Epidemiología, Instituto de Medicina Tropical, Facultad de Medicina, Universidad Nacional Mayor de San Marcos. Lima, Perú.
}

\begin{abstract}
Resumen
Introducción: El curso de Epidemiología se dicta en el $5^{\circ}$ año de pregrado de la Escuela Académico Profesional de Medicina Humana de la Facultad de Medicina de la Universidad Nacional Mayor de San Marcos (UNMSM). Uno de los productos finales del curso es la realización de un trabajo de investigación. Objetivos: Determinar el porcentaje de trabajos de investigación producidos durante el curso de Epidemiologia y que son publicados en revistas biomédicas durante el periodo 2003 y 2009. Diseño: Estudio bibliométrico. Lugar: Sección de Epidemiología del Instituto de Medicina Tropical, Universidad Nacional Mayor de San Marcos. Material bibliográfico: Trabajos de investigación publicados. Métodos: Número de trabajos de investigación producidos en el curso de Epidemiología en el periodo 2003-2009 y publicados en revistas biomédicas. Principales medidas de resultados: Número de trabajos de investigación producidos y publicados en revistas biomédicas. Resultados: Se produjeron 110 trabajos de investigación, de los cuales $13(11,8 \%)$ fueron publicados en alguna revista biomédica. El número promedio de autores de los trabajos publicados fue de siete estudiantes. En ocho de los trabajos publicados se incluyó al profesor asesor como autor. Conclusiones: La producción de publicaciones estudiantiles es baja, la falta de interés por publicar de los médicos peruanos se observa desde el pregrado. Existen factores asociados al estudiante, al plan de estudio y a las mismas revistas biomédicas que podrían explicar este fenómeno.

Palabras clave: Estudiantes de medicina; informes de investigación; publicaciones científicas y técnicas.
\end{abstract}

\begin{abstract}
Background: The epidemiology course is taught in the 5th year of undergraduate Academic Professional School of Human Medicine, Faculty of Medicine of the San Marcos University. One of the outcomes at the end of this course is research that usually will not be published. Objectives: To determine the percentage of research produced during the course of epidemiology and published in biomedical journals during the period 2003-2009. Design: Bibliometric study. Setting: Epidemiology Section, Institute of Tropical Medicine, Universidad Nacional mayor de San Marcos, Lima, Peru. Bibliographic material: Research work published. Methods: Papers elaborated in the epidemiology course and published in biomedical journals during the period 2003-2009. Main outcome measures: Number of research papers produced and published in biomedical journals. Results: From 110 research works $13(11.8 \%)$ were published in biomedical journals. Authors average number per paper was 7 students; 8 papers considered the tutor as an author. Conclusions: The production of student publications is low. Peruvian physicians' lack of interest in publishing is observed since undergraduate. There are factors associated with the student, study plans and biomedical journals that could explain this phenomenon.
\end{abstract}

Key words: Students, medical; research reports; scientific and technical publications.

\section{INTRODUCCIÓN}

La epidemiología es el estudio de cómo las enfermedades se distribuyen en las poblaciones y de los factores que influencian o determinan su distribución ${ }^{(1,2)}$. Otra definición más precisa nos dice que la epidemiología es la ciencia que estudia las causas de la aparición, propagación y mantenimiento de los daños a la salud en las poblaciones, con la finalidad de prevenirlos o controlarlos ${ }^{(3)}$. Esta ciencia es parte básica e importante en la formación del futuro médico, por lo que su enseñanza a través de la asignatura de Epidemiología es dirigida a los estudiantes del quinto año de pre-grado como parte del plan de estudios de la Escuela Académico Profesional de Medicina Humana de la Facultad de Medicina de la Universidad Nacional Mayor de San Marcos (UNMSM). Este curso es importante en la formación clínica, ya que aporta conocimientos para la descripción del proceso de propagación de enfermedades, haciendo énfasis en el establecimiento de relaciones causales así como el estudio, diseño y ejecución de intervenciones de prevención y control de enfermedades. También proporciona instrumentos para el análisis crítico de los resultados de los estudios epidemiológicos y el desarrollo de investigaciones epidemiológicas; brinda además conocimientos acerca del perfil epidemiológico del Perú y enfatiza en la aplicación de los principios morales que garanticen el desarrollo de una investigación ética (4).
Entre las competencias de este curso se encuentra el capacitar al alumno para ejecutar estudios epidemiológicos, desde el diseño hasta la presentación del informe; es decir, se incentiva que el alumno se inicie en la investigación como parte de su formación integral como médico. Uno de los productos finales del curso es la presentación de un estudio de investigación realizado por los alumnos y asesorado por un docente ${ }^{(4)}$. El proceso de desarrollo del estudio de investigación en el curso se realiza mediante siete gabinetes donde se cumple los siguientes pasos: selección del tema y formulación del problema a investigar, búsqueda bibliográfica, elaboración del diseño de investigación, selección de la población de estudio, prueba piloto, ejecución del trabajo de campo, análisis 
de resultados, discusión de los resultados obtenidos y preparación del informe final (producto) ${ }^{(5)}$. A pesar de que el curso incentiva la publicación de estos estudios mediante la difusión de trabajos hechos previamente y que han sido publicados en revistas biomédicas o mediante el estimulo en los talleres prácticos, muchos de estos trabajos de investigación no llegan a ser publicados en revistas científicas biomédicas, o sus resultados no se llegan a difundir en los diferentes congresos de estudiantes de medicina o jornadas estudiantiles. Este hecho es compatible con la llamada "Pirámide de producción científica nacional", que podría aplicarse para la investigación en pregrado, ya que son muchos los estudios científicos realizados en varios de los cursos; sin embargo, pocos son publicados o expuestos en jornadas o congresos científicos ${ }^{(6)}$.

Un estudio sobre la contribución de estudiantes de medicina en publicaciones científicas refleja que los estudiantes participaron en $4,5 \%$ del total de las publicaciones ${ }^{(7)}$. Un porcentaje similar de aporte se observa en la publicación estudiantil de reportes y series de casos (8). Según los libros de resúmenes de los Congresos Científicos Nacionales organizados por la Sociedad Científica Médica Estudiantil Peruana (SOCIMEP), se realizaron, entre 1993 y 2003, 679 trabajos de investigación. De estos, 52\% consistió en investigaciones sobre salud pública ${ }^{(9)}$.

Existen algunas debilidades en la publicación científica en el pregrado, como el plagio. Un estudio encontró que 95,8\% de las publicaciones realizadas por estudiantes tuvieron alguna forma de copia electrónica. La falta de entrenamiento en la búsqueda bibliográfica se refleja en que solo $29,5 \%$ de publicaciones usó referencias provenientes de publicaciones científicas para configurar el proyecto de investigación y por ende el artículo científico ${ }^{(10)}$. Gutiérrez y col., en su revisión sobre publicación en pregrado, mencionan como dificultades para publicar en pregrado la falta de valoración del trabajo realizado, ausencia de cultura de publicación, falta de capacitación, desconocimiento de oportunidades, percepción de calidad pobre de la investigación, poco apoyo docente y falta de incentivos ${ }^{(11)}$. Estas debilidades y dificultades junto a la falta de interés de los estudiantes de pregrado en la investigación hacen que la participación en todos los tipos de publicación sea menor de 5\%.

Según Gómez y col. existen cuatro formas de realizar investigación en el pregrado: iniciativa propia de estudiantes agrupados en sociedades científicas, invitación para participar en un proyecto propuesto por un investigador principal, investigación como parte de un curso -por ejemplo metodología de investigación, estadística, farmacología o epidemiología- e investigación en el marco de la realización de la tesis de grado ${ }^{(12)}$.

Centrándonos en la investigación en pregrado como parte de un curso, tenemos que el curso de Epidemiología es uno de los que brinda espacio y tiempo para desarrollar investigación estudiantil en la carrera de medicina humana. En la facultad de medicina se incluye cursos de investigación, los cuales no llegan a cubrir el 10\% del total de créditos del programa de medicina ${ }^{(7)}$; además, existen otros cursos en los cuales se requiere de un trabajo de investigación para completar con el creditaje del curso (por ejemplo Parasitología, Farmacología, Patología, Ginecología y Obstetricia) (figura 1).

En el presente trabajo describiremos la producción de trabajos de investigación publicados en revistas biomédicas que han sido elaborados durante el curso de Epidemiología, en el periodo 2003 - 2009. Además, revisaremos los factores asociados a la publicación de estos trabajos de investigación y la importancia de porqué deberían ser publicados estos trabajos.

\section{MÉTODOS}

Se realizó un estudio bibliométrico. Se utilizó los archivos internos del curso de Epidemiología, en donde se encuentran registrados los trabajos de investigación producidos entre los años 2003 y 2009 y que han sido posteriormente publicados en revistas biomédicas. Luego, se procedió a la búsqueda de dichos trabajos de investigación (artículos originales) publicados en la versión online de la revista correspondiente.

El número total de trabajos de investigación realizados durante dicho curso por año es equivalente al número de grupos de práctica formados para dicho año. Dicho dato se obtuvo de los sílabos del curso de epidemiología, a partir del año 2003.

El porcentaje de trabajos de investigación publicados fue calculado para todo el periodo de análisis, de la siguiente manera:

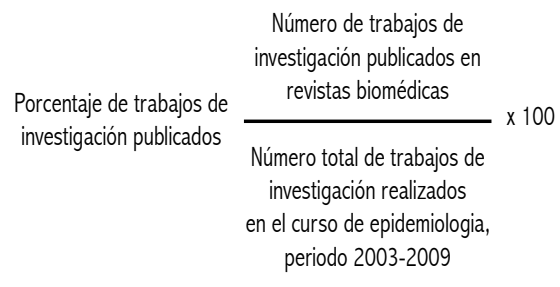

El número de trabajos de investigación realizados durante el periodo de estudio fue 110. El número de trabajos varió por año, dependiendo del número de alumnos de la promoción ingresante. De esta forma, el año 2003 se realizó 18 trabajos; el año 2004, 24 trabajos; el año 2005, 24 trabajos; el año 2006, 12 trabajos; el año 2007, 12 trabajos; el año 2008, 10 trabajos; y el año 2009, otros 10 trabajos. La identificación de los trabajos de investigación que ingresaron al estudio se hizo observando la filiación del autor e identificando a los autores como alumnos del curso de epidemiología en los registros del curso. El análisis de datos fue descriptivo. Los resultados se presenta como frecuencias y porcentajes.

\section{RESULTADOS}

Entre el año 2003 y 2009 se formaron 110 grupos de práctica para el curso de Epidemiología, los cuales luego de la realización de gabinetes de trabajo produjeron 110 trabajos de investigación. De los 110 trabajos de investigación, 13 fueron publicados en revistas biomédicas, siendo $11,8 \%$ el porcentaje de trabajos de investigación que fue publicado como artículo original en alguna revista biomédica. Los 13 trabajos fueron publicados en revistas biomédicas nacionales y el resumen de uno de ellos, en una revista internacional. En promedio, se ha publicado en revistas biomédicas menos de dos trabajos de investigación por año.

Casi el $40 \%$ de los trabajos publicados fue realizado en el año 2005. El número promedio de autores por artículo fue de siete estudiantes, con un mínimo de cuatro y máximo de 11 estudiantes. Cinco 


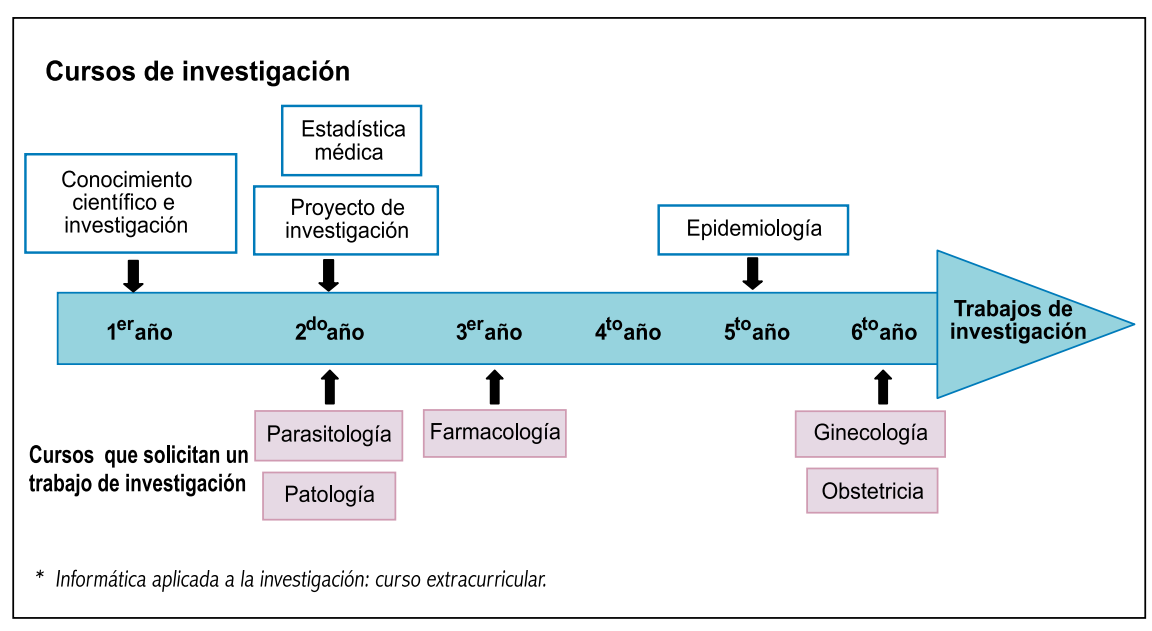

Figura 1. Plan de estudios de la Escuela Académico Profesional de Medicina Humana de la UNMSM, en relación a la investigación en pregrado.

de los 13 trabajos originales publicados tuvieron de diez a más autores.

En ocho de 13 (61,5\%) trabajos publicados en revistas biomédicas, el profesor asesor del proyecto también fue incluido como autor del trabajo publicado.

Dos de los trabajos fueron publicados en una revista de Dermatología, otros tres en una revista de Salud Pública; seis artículos fueron publicados en revistas especializadas en Epidemiología, dos artículos en la revista de la facultad de medicina de la UNMSM y un trabajo en una revista estudiantil.

En la tabla se muestra los trabajos de investigación publicados durante los años analizados y que fueron realizados en el curso de epidemiología.

\section{DISCUSIÓN}

En el presente análisis, se encontró que 110 trabajos de investigación fueron realizados entre los años 2003 y 2009, en el curso de Epidemiología. Solo el $11,8 \%$ de estos trabajos de investigación fue publicado como artículo original en alguna revista biomédica.

La producción de artículos publicados no fue homogénea durante cada uno de los años estudiados. Esto estuvo determinado en parte por la cantidad de trabajos de investigación realizados que a su vez dependía del número de alumnos que llevaron el curso en el año y de otros factores, que posteriormente discutiremos.
La poca producción estudiantil de trabajos de investigación es un fenómeno que ya ha sido explorado en estudios nacionales y extranjeros. Un estudio comunica que, en el periodo 1993 al 2003, se presentó a los congresos científicos nacionales un promedio de 60 trabajos por año; 8 de estos trabajos, una pequeña proporción llegó a ser publicado. Varios estudios tratan de explicar el porqué existe una baja producción de trabajos publicados ${ }^{(7,11,13)}$; sin embargo, menos trabajos exploran porqué no se realizan trabajos de investigación ${ }^{(14)}$, sin considerar que el primer paso para publicar es el investigar. Un interesante estudio refiere que el autoconocimiento de deficiencias metodológicas es una de las principales razones que expresan los alumnos de pregrado para no investigar. Otras razones referidas por los alumnos son la falta de tiempo y de recursos económicos. Mientras que casi $20 \%$ de dichos alumnos refiere no tener gusto por la investigación ${ }^{(14)}$.

El aporte estudiantil en la publicación de artículos en revistas médicas peruanas no llega al $5 \%{ }^{(7)}$; lo mismo pasa con el aporte de estudiantes de medicina en la publicación de comunicaciones de casos, el cual llega al $4,8 \%{ }^{(8)}$. Se ha publicado dos estudios acerca de la publicación de tesis realizadas por estudiantes de medicina. Entre los años 2000 y 2003, 482 tesis médicas fueron registradas en la Universidad Peruana Cayetano Heredia, de las cuales $85(17,6 \%)$ fueron publicadas en revistas biomédicas indizadas ${ }^{(15)}$. Un estudio similar realizado en la Universidad Nacional
Mayor de San Marcos indica que, entre 1998 y 2008, se presentaron 93 tesis, de las cuales solo $11(11,8 \%)$ fueron publicadas en una revista biomédica indizada ${ }^{(16)}$. Es decir, nuestros resultados son iguales a la tasa de publicación de tesis médicas producidas por los estudiantes de San Marcos. Sin embargo, debería ser preocupante para la universidad que en un periodo de 11 años solo se hayan producido 93 tesis en pregrado, mientras que es estimulante para el curso de epidemiologia que en un periodo menor (siete años) solo en dicho curso se hayan producido 110 trabajos de investigación.

Como vemos, la tasa de publicación de trabajos de investigación en pregrado varía entre $11,8 \%$ y $17,6 \%$. Así, la producción científica de estudiantes de medicina sigue la 'pirámide de la producción científica nacional', ya que como parte de muchos cursos se realizan proyectos de investigación. Sin embargo, son pocos los cursos en los cuales se tiene que realizar tanto el proyecto como el trabajo de investigación, obteniendo resultados; de estos trabajos de investigación, solo una minoría llega a ser publicada en revistas biomédicas ${ }^{(6)}$. (Figura 2).

Como sabemos, la redacción de un artículo científico involucra una serie de procesos que requieren habilidades, competencias y conocimientos. Esta etapa es esencial en el proceso de investigación. La investigación no culmina con la producción de resultados; esta requiere la publicación de dichos resultados ${ }^{(11)}$.

Factores implicados en la publicación de trabajos de investigación

Existe una serie de factores que determinarían la publicación de trabajos de investigación, los cuales podrían ser agrupados como factores relacionados al estudiante, al plan de estudios y a las revistas biomédicas. Los factores implicados en la publicación no son los mismos a los relacionados a la realización de trabajos de investigación.

Factores asociados al estudiante y al mismo trabajo de investigación

Dentro de estos factores podemos citar: falta de valoración del trabajo realizado $(11,13,14,17,18)$, ausencia de cultura de publicación ${ }^{(6,11,13)}$, desconocimiento de oportunidades para publicar ${ }^{(11)}$, calidad 
Tabla. Trabajos de investigación realizados en el curso de epidemiología y publicados en revistas biomédicas

\begin{tabular}{|c|c|c|c|c|}
\hline Autor principal & Título del trabajo & Revista (año); Volumen $\left(\mathrm{N}^{\circ}\right.$ ) & $\begin{array}{l}\text { Año de realización* } \\
\text { del estudio }\end{array}$ & $\begin{array}{l}\text { Número de } \\
\text { autores }\end{array}$ \\
\hline Hugo Cajaleón & $\begin{array}{l}\text { Supervivencia en pacientes afectados por melanoma } \\
\text { maligno en el Instituto Nacional de Enfermedades } \\
\text { Neoplásicas. }\end{array}$ & Folia Dermatológica Peruana. 2005;16(1) & 2003-I & 5 \\
\hline Franco Romaní & $\begin{array}{l}\text { Conocimientos, actitudes y prácticas sobre protección } \\
\text { solar en Internos de Medicina de cinco hospitales } \\
\text { generales de Lima y Callao. }\end{array}$ & Folia Dermatológica Peruana. 2005;16(2) & $2005-11$ & 11 \\
\hline José Juárez & $\begin{array}{l}\text { Evaluación de la definición de caso probable de dengue } \\
\text { clásico durante el brote de dengue en Lima, } 2005 .\end{array}$ & $\begin{array}{l}\text { Revista Peruana de Medicina Experimental } \\
\text { y Salud Pública. 2005;22(3) }\end{array}$ & $2005-1$ & 6 \\
\hline Jerson Muñoz & $\begin{array}{l}\text { Suicidal ideation and family cohesion y pre-college } \\
\text { students, Lima-Peru } 2005 .\end{array}$ & $\begin{array}{l}\text { American Journal of Epidemiology. } \\
\text { Abstract 2006;163(Supl) }\end{array}$ & $2005-11$ & 4 \\
\hline Jerson Muñoz & $\begin{array}{l}\text { Ideación suicida y cohesión familiar en estudiantes } \\
\text { preuniversitarios entre } 15 \text { y } 24 \text { años, Lima. } 2005 .\end{array}$ & $\begin{array}{l}\text { Revista Peruana de Medicina Experimental } \\
\text { y Salud Pública. 2006;23(4) }\end{array}$ & $2005-11$ & 4 \\
\hline Rubén Valle & $\begin{array}{l}\text { Sintomatología depresiva y calidad de vida en } \\
\text { pacientes mujeres con cáncer de mama. }\end{array}$ & $\begin{array}{l}\text { Anales de la Facultad de Medicina de la } \\
\text { UNMSM. 2006;67(4) }\end{array}$ & $2006-\mid$ & 5 \\
\hline Juan Astuvilca & $\begin{array}{l}\text { Incidencia y factores asociados con las reacciones } \\
\text { adversas del tratamiento antirretroviral inicial en } \\
\text { pacientes con VIH. }\end{array}$ & $\begin{array}{l}\text { Revista Peruana de Medicina Experimental } \\
\text { y Salud Pública. 2007;24(3) }\end{array}$ & $2007-\mid$ & 7 \\
\hline Pilar Aramburú & $\begin{array}{l}\text { Prevalencia y factores asociados a depresión posparto } \\
\text { en mujeres atendidas en establecimiento de salud del } \\
\text { primer nivel de atención en Lima Metropolitana, junio } 2004 .\end{array}$ & $\begin{array}{l}\text { Revista Peruana de Epidemiología. } \\
2008 ; 12(3)\end{array}$ & 2004-I & 6 \\
\hline María Yengle & $\begin{array}{l}\text { Prácticas de utilización de plaguicidas en agricultores en } \\
\text { el distrito de Huaral - Perú. Noviembre } 2005 .\end{array}$ & $\begin{array}{l}\text { Revista Peruana de Epidemiología. } \\
2008 ; 12(1)\end{array}$ & 2005-I & 10 \\
\hline Cindy Alcarraz & $\begin{array}{l}\text { Factores asociados a realización de prueba de detección } \\
\text { para VIH en estudiantes universitarios de Lima. }\end{array}$ & CIMEL. 2008;13(2) & 2008-1 & 5 \\
\hline Paolo Wong & $\begin{array}{l}\text { Caracterización de los accidentes de tránsito en la } \\
\text { región Callao-Perú, 1996-2004. }\end{array}$ & $\begin{array}{l}\text { Revista Peruana de Epidemiología. } \\
2009 ; 13(3)\end{array}$ & $2005-11$ & 10 \\
\hline Oscar Alvis & $\begin{array}{l}\text { Factores asociados a la no adherencia al tratamiento } \\
\text { antirretroviral de gran actividad en adultos infectados } \\
\text { con el VIH-SIDA. }\end{array}$ & $\begin{array}{l}\text { Anales de la Facultad de Medicina de la } \\
\text { UNMSM. 2009;70(4) }\end{array}$ & $2007-\mid$ & 5 \\
\hline Katia Bravo & $\begin{array}{l}\text { Nivel de conocimientos sobre la influenza AH1N1 en los } \\
\text { trabajadores del Hospital Nacional Arzobispo Loayza, Perú. }\end{array}$ & $\begin{array}{l}\text { Revista Peruana de Epidemiología. } \\
\text { 2009:13(2) }\end{array}$ & 2009-I & 11 \\
\hline Jonny Laurente & $\begin{array}{l}\text { Conocimientos y actitud acerca de la transmisión y } \\
\text { prevención de la TBC en pacientes con tuberculosis } \\
\text { multidrogoresistente. }\end{array}$ & $\begin{array}{l}\text { Revista Peruana de Epidemiología. } \\
2010 ; 14(1)\end{array}$ & - & 10 \\
\hline
\end{tabular}

* Se considera el año y el semestre en el cual se realizó el estudio (I de marzo a julio y Il de agosto a diciembre).

intrínseca de la investigación ${ }^{(11)}$, falta de tiempo ${ }^{(11,13)}$, debilidades en la redacción científica, desconocimiento sobre en qué revistas publicar ${ }^{(7,13)}$, desconocimiento del proceso de envío de trabajos a una revista ${ }^{(7)}$, falta de interés por la investigación y publicación ${ }^{(14,17)}$, falta de dominio del inglés para publicar en revistas extranjeras ${ }^{(13)}$ y falta de actitudes positivas frente a investigación científica en medicina ${ }^{(18,19)}$.

Los cambios en la actitud de los estudiantes de medicina a favor de la investigación y publicación no pueden darse rápidamente. Según Marusic y col., el objetivo a largo plazo es crear una masa de médicos académicos con habilidades críticas necesarias no solo para la medicina basada en evidencia sino también para plantearse preguntas de investigación ${ }^{(20)}$.

Un estudio realizado en la Escuela de Medicina Mayo informa que 41\% de sus graduados había publicado uno o más trabajos de investigación y $18 \%$ había publicado uno o más resúmenes (21) . Para lograr esta alta productividad en publicaciones es necesario concientizar a los estudiantes de medicina de la importancia del proceso de investigación para un médico actual. Sin embargo, los cambios también deben darse a nivel curricular, para brindar a los alumnos las competencias y habilidades para una investigación seria y rigurosa.

Factores asociados al plan de estudio y docentes

Dentro de este grupo podemos citar: falta de curso o capítulo de redacción científica (13), falta de motivación docente por la publicación ${ }^{(11,13,22)}$, falta de incentivo a nivel académico ${ }^{(13)}$, limitación en el análisis de datos ${ }^{(13)}$, inadecuada instrucción en el proceso de publicación (7), falta de contactos y coordinación de los 


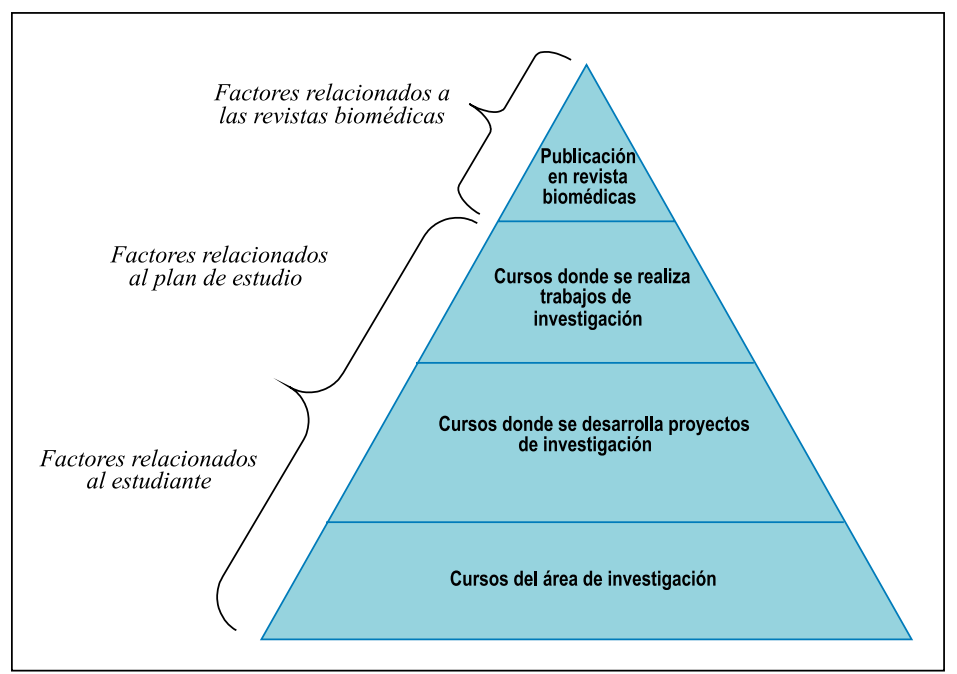

Figura 2. Pirámide de producción científica en los cursos de pregrado.

cursos con equipos editoriales de revistas biomédicas.

Un aspecto importante sugerido por Gutiérrez y col. es pedir a las universidades que dentro de los cursos donde se hace investigación se haga especial énfasis en la publicación de dichos trabajos ${ }^{(11)}$; de esta forma se logra una mayor difusión que la lograda en la simple exposición al final del curso, en jornadas científico estudiantiles o en los libros de resúmenes ${ }^{(6)}$.

Factores asociados a las revistas biomédicas

Dentro de este grupo de factores tenemos el escaso número de revistas biomédicas exclusivas para estudiantes de medicina (Revista CIMEL) ${ }^{22)}$, poco número de revistas editadas por universidades (Anales de la Facultad de Medicina y Revista Médica Herediana), percepción de los estudiantes de medicina de que las revistas biomédicas no publican trabajos de estudiantes, falta de realización de la labor de captación de trabajos de investigación por parte de las revistas biomédicas.

Un aspecto importante es el antecedente de publicación de trabajos de estudiantes en una determinada revista, ya que este antecedente es un llamado a los estudiantes que buscan dónde publicar y encuentran en dicha revista un potencial espacio para publicar.

Importancia de la publicación de trabajos de investigación en pregrado

La publicación de trabajos de investigación realizados en pregrado es importante para todos los actores involucrados: estudiantes, docentes, plan curricular y universidad.

- Fomenta la actividad de investigación en todas sus etapas hasta la publicación, ya que la finalidad de la investigación es la producción de nuevo conocimiento, el cual debe difundirse mediante la publicación. De esta forma, la publicación estudiantil contribuiría a la producción científica nacional ${ }^{(13,22)}$. Otro aspecto importante es que la difusión de los resultados permite la retroalimentación (feedback), lo cual también aporta a la investigación realizada aún si esta ya está publicada, ya que el problema inicial puede tener nuevos puntos de vista, el tema puede ser profundizado o se puede buscar soluciones o aplicaciones prácticas a los resultados obtenidos ${ }^{(11)}$.

- Fomenta la cultura de investigación, ya que al investigar y publicar desde el pregrado se va formando un hábito y actitud favorable frente a la investigación y publicación; esto hace que los médicos se familiaricen con el proceso de publicación, adquiriendo experiencia en la redacción, llegando a ser la publicación e investigación parte importante y cotidiana de la práctica médica continua (11,13,14,22). Esta cultura de investigación debe tener la capacidad de estimular tanto a alumnos como a docentes en la publicación y debe ser bidireccional (alumno-docente, docente-alumno). Un aspecto importante de la investigación es el desarrollo de la capacidad crítica de la literatura biomédica; esta habilidad también es desarrollada a medida el alumno investigue y publique, mejorando progresivamente la calidad de su búsqueda bibliográfica y análisis crítico de esta literatura, que se ve expresada en cada vez mejores discusiones y presentación de resultados ${ }^{(11,13)}$.

- Permite una valoración personal y reconocimiento como investigador. Considerando que la metodología de investigación fue rigurosa, adecuada y ética, los estudiantes no deberían desestimar sus trabajos como publicables por el hecho de ser aún estudiante ${ }^{(11,13)}$. El publicar deja huella en la literatura biomédica y el valor de dicha publicación -y por ende el reconocimiento como investigador- será obtenido de la valoración individual o colectiva del lector o comunidad médica, respectivamente. Este reconocimiento va creando una carta de presentación, sobre todo si existe calidad en lo que se publica. La publicación es el mejor indicador de evaluar la productividad científica: sin embargo, el aspecto cualitativo debe ser asegurado en la medida de lo posible, mediante el uso del sistema de revisión por pares ${ }^{(11,13)}$.

Como vemos, la importancia de la publicación en pregrado es evidente y sustentable por muchos motivos. Sin embargo, la realidad indica que aún no se ha establecido mecanismos necesarios para mejorar la productividad científica en pregrado o, si han sido establecidos, aún no se hace manifiesta la mejora en la tasa de publicación.

En conclusión, a pesar de que existen (23) facultades de medicina en el país, el porcentaje de trabajos originales publicados por todos los estudiantes de medicina de estas facultades no llega al 5\%. En la presente comunicación analizamos la producción de publicaciones de un curso de pregrado de la Universidad Nacional Mayor de San Marcos. Según este análisis, solo $11,8 \%$ de los trabajos de investigación se publica. Así, a pesar que se realizan trabajos de investigación en varios 
cursos del pregrado, este porcentaje sería un indicador aproximado de la cantidad de trabajos que llegan a ser publicados. Hemos planteados factores para explicar este fenómeno denominado 'pirámide de publicación científica nacional', con el fin de determinar las variables que pueden ser corregidas a mediano y largo plazo, con el objetivo de cambiar el perfil del graduado en medicina y concientizar sobre el concepto universal de que la investigación es un proceso que culmina con la publicación del manuscrito, y que dicha publicación es tan valiosa al ser hecha como estudiante como al momento de ser médico especializado en alguna rama de la medicina.

\section{Recomendaciones}

Muchas recomendaciones respecto al aporte estudiantil en las revistas biomédicas han sido dadas ${ }^{(6,7,9,11,14)}$; sin embargo, queremos puntualizar en las siguientes:

- Establecer mecanismos por el cual las publicaciones estudiantiles sean valoradas de alguna manera (puntuación para postulación a especialidades y puestos de trabajo), tomando en cuenta el factor de impacto de la revista biomédica.

- Establecer mecanismos por el cual se publiquen los mejores trabajos de investigación realizados en cada uno de los cursos para su publicación.

- Mejorar la enseñanza en cada uno de los cursos de investigación, incorporando en el estudiante actitudes, competencias y habilidades necesarias para desarrollar investigación rigurosa y publicable.

- Establecer líneas de investigación que surjan de los propios cursos de pre-grado, las cuales deberían ser respaldadas por los institutos de investigación de la universidad.

\section{REFERENCIAS BIBLIOGRÁFICAS}

1. Gordis L (ed). Epidemiology: Introduction. 3ra edición. Philadelphia: WB Saunders Company; 2006. p. 3.
2. Weiss N (ed). Clinical Epidemiology: What it is and how it is used?. New York: Oxford University Press; 1996. p. 3.

3. Alarcón J. Epidemiología general: Concepto, enfoques, dinámicas y usos de la epidemiología. Lima: Facultad de Medicina, UNMSM; 2007. p. 5-10. Disponible en: http://www.epiredperu. net/epired/cursos/epidemiologia_bas-mh/ epibas_text01.pdf

4. Facultad de Medicina, UNMSM. Curso de epidemiología básica. Año académico 2009 I. Lima: Facultad de Medicina, UNMSM; 2009. Disponible en: http://www.epiredperu.net/epired/ cur_epidemiologia_bas-mh-09l.html

5. Facultad de Medicina, UNMSM. Sillabus de Epidemiología. Año académico 2009-I. Escuela Académico Profesional de Medicina humana. Departamento académico de medicina preventiva y salud pública. Lima: Facultad de Medicina, UNMSM; 2009. Disponible en: http://www. epiredperu.net/epired/cursos/epidemiologia bas-mh/epibas_syl_09-I.pdf

6. Pamo 0. Estado actual de las publicaciones periódicas científicas médicas del Perú. Rev Med Hered. 2005;16(1):65-73. Disponible en: http:// www.scielo.org.pe/pdf/rmh/v16n1/v16n1ce1.pdf

7. Huamaní Ch, Chávez P, Mayta P. Aporte estudiantil en la publicación de artículos científicos en revistas médicas indizadas en SciELO-Perú, 1997- 2005. An Fac med. 2008;69(1):42-5. Disponible en: http://sisbib.unmsm.edu.pe/BVre vistas/anales/ v69n1/pdf/a09v69n1.pdf

8. Romaní F, Wong P. Reporte y serie de casos en el Perú: situación de un tipo de publicación subestimada. Análisis de las revistas médicas peruanas indizadas en SciELO-Perú, 1997-2008. Rev peru epidemiol [Internet]. 2009 [citada el 15 febrero de 2010];13(3): [aprox. 7 p.]. Disponible en: http://rpe.epiredperu.net/rpe_ediciones/2009_ v13_n03/A07_Vol13_No3_2009_Reporte\%20 y\%20series\%20de\%20caso.pdf

9. Galán E, Manrique N, Villavicencio E, Yllatopa E, Peralta M, De la Cruz W. Producción científica de los investigadores del pregrado de medicina humana del Perú, 1993-2003. CIMEL. 2005; 10(1):41-8.

10. Huamaní Ch, Dulanto A, Rojas V. 'Copiar y pegar' en investigaciones en el pregrado: haciendo mal uso del Internet. An Fac med. 2008;69(2):117-9.

11. Gutiérrez C, Mayta P. Publicación desde el pregrado en Latinoamérica: Importancia, limitaciones y alternativas de solución. CIMEL. 2003;8(1):5460.

12. Gómez R, Herrera A. Diagnóstico de la investigación en los estudiantes de pregrado en ciencias de la salud en Risaralda. Rev Med Risaralda. 2002;8(2):32-8.

13. Molina J, Huamaní Ch, Mayta P. Apreciación estudiantil sobre la capacitación universitaria en investigación: estudio preliminar. Rev Peru Med Exp Salud Pública. 2008;25(3):325-9.

14. Ramos M, Sotomayo R. Realizar o no una tesis: razones de estudiantes de medicina de una universidad pública y factores asociados. Rev Peru Med Exp Salud Pública. 2008;25(3): 322-4.

15. Arriola I, Curioso W, Cruz M, Gayoso 0. Characteristics and publication patterns of theses from a Peruvian medical school. Health information and libraries journal. 2010;27(2):148-54.

16. Valle R, Salvador E. Análisis bibliométrico de las tesis de pregrado de la Facultad de Medicina de la Universidad Nacional Mayor de San Marcos. An Fac med. 2009;70(1):11-8.

17. Jacobs Ch, Cross P. The value of medical student research: the experience at Stanford University School of Medicine. Medical Education 1995;29:342-6.

18. Pruskil S, Burgwinkel P, Georg W, Keil T, Kiessling C. Medical students' attitudes towards science and involvement in research activities: A comparative study with students from a reformed and a traditional curriculum. Medical Teacher. 2009;31:e254-9.

19. Hren D, Lukic IK, Marusic A, Vodopivec I, Vujaklija A, Hrabak M, et al. Teaching research methodology in medical schools: students' attitudes towards and knowledge about science. Med Educ. 2004;38(1):81-6.

20. Marusic A, Marusic M. Teaching students how to read and write science: a mandatory course on scientific research and communication in medicine. Acad Med. 2003;78:1235-9.

21. Dyrbye L, Davidson L, Cook D. Publications and presentations resulting from required research by students at Mayo Medical School, 1976-2003. Acad Med. 2008;83:604-10.

22. Mayta P, Peña A. Importancia de la publicación en las sociedades científicas de estudiantes de medicina del Perú: estudio preliminar. CIMEL 2009;14(1):27-34.

Fuente de financiamiento: Trabajo autofinanciado.

Manuscrito recibido el 10 de mayo de 2010 y aceptado para publicación el 10 de junio de 2010.

\section{Correspondencia:}

Jorge Alarcón Villaverde

Instituto de Medicina Tropical "Daniel A. Carrión"

Facultad de Medicina, UNMSM.

Jr. José Santos Chocano 199. 4to. piso

Urb. San Joaquín - Bellavista

Callao 2, Perú.

Correo electrónico: jalarconv@epiredperu.net 